\title{
Classification of damage in structural systems using time series analysis and supervised and unsupervised pattern recognition techniques
}

\author{
Piotr Omenzetter*, Oliver R. de Lautour \\ Department of Civil and Environmental Engineering, The University of Auckland, Private Bag \\ 92019, Auckland Mail Centre, Auckland 1142, New Zealand
}

\begin{abstract}
Developed for studying long, periodic records of various measured quantities, time series analysis methods are inherently suited and offer interesting possibilities for Structural Health Monitoring (SHM) applications. However, their use in SHM can still be regarded as an emerging application and deserves more studies. In this research, Autoregressive (AR) models were used to fit experimental acceleration time histories from two experimental structural systems, a 3storey bookshelf-type laboratory structure and the ASCE Phase II SHM Benchmark Structure, in healthy and several damaged states. The coefficients of the AR models were chosen as damage sensitive features. Preliminary visual inspection of the large, multidimensional sets of AR coefficients to check the presence of clusters corresponding to different damage severities was achieved using Sammon mapping - an efficient nonlinear data compression technique. Systematic classification of damage into states based on the analysis of the AR coefficients was achieved using two supervised classification techniques: Nearest Neighbor Classification (NNC) and Learning Vector Quantization (LVQ), and one unsupervised technique: Self-organizing Maps (SOM). This paper discusses the performance of AR coefficients as damage sensitive features and compares the efficiency of the three classification techniques using experimental data.
\end{abstract}

Keywords: Structural Health Monitoring, damage detection, Autoregressive models, time series analysis, supervised learning, unsupervised learning, Nearest Neighbor Classification, Learning Vector Quantization, Self-organizing Maps

\section{INTRODUCTION}

Time series techniques, originally developed for analyzing long sequences of regularly sampled data, are inherently suited to Structural Health Monitoring (SHM). However, such techniques have yet to be fully explored. Fassois and Sakellariou ${ }^{1}$ present an overview of the principles and techniques of time series analysis and their classification into nonparametric and parametric methods for applications in SHM. Differentiation between faulty or damaged and healthy systems was achieved within the framework of statistical hypothesis testing. As the present study uses a parametric method, the following literature review focuses mainly on such approaches and on investigations where the coefficients of time series models were used to form damage sensitive features. Sadeghi and Fassois ${ }^{2}$ applied Autoregressive Moving Average models with eXogenous input (ARMAX) to dynamic signals from a laboratory beam and a numerical model of a truss and used coefficients of those models as damage sensitive features. Damage was detected and broadly localized by locating the feature vectors in the hyperspace spanned by their means and standard deviations. Feature dimensionality reduction via Principal Component Analysis (PCA) and an information entropy based criterion was also studied. Sakellariou and Fassois ${ }^{3}$ extended the approach by observing changes in time series coefficients and used them for damage detection, localization and severity estimation in a numerical, 6-DOF, linear model of a shear type building subjected to seismic excitation. Sakellariou and Fassois ${ }^{4}$ generalized the method using Autoregressive models with eXogenous input (ARX) whose coefficients were functions of fault magnitude and applied it to study damage in a laboratory aircraft skeleton structure. A study by Sohn et al. ${ }^{5}$ used Autoregressive (AR) models to fit the dynamic response of a concrete bridge pier. By applying a statistical process control approach to the projected coefficients of the AR models the authors were able to distinguish between healthy and damaged states. A later study by Sohn et al. ${ }^{6}$ applied a similar methodology to health monitoring of a surface-effect fast patrol boat. However, the authors did not attempt to locate or quantify damage. Gul and Catbas ${ }^{7}$ presented a study in which AR coefficients from a laboratory steel

"*omenzetter@auckland.ac.nz; phone $6493737-599$ ext. 88138; fax 64 93737-462; auckland.ac.nz 
beam and grid structure with varying support and connectivity conditions were classified using a multivariate outlier detection technique. Omenzetter and Brownjohn ${ }^{8}$ used a vector Seasonal Autoregressive Integrated Moving Average model to detect abrupt changes in strain data collected from the continuous monitoring of a major bridge structure. The seasonal model was used because of the strong diurnal variation in the data caused by the temperature cycle. Zheng and Mita $^{9-11}$ used Autoregressive Moving Average (ARMA) models in a two-stage damage assessment method where differences between ARMA models were calculated as their Itakura distances, model cepstra or subspace angle distances used as damage detection features, and enhanced localization performance was achieved using pre-whitening filters. The approaches were applied to numerically simulated and experimental laboratory data. Nair et al. ${ }^{12}$ used an ARMA time series to model the vibration signals from the ASCE Phase II Experimental SHM Benchmark Structure. The authors defined a damage sensitive feature used to discriminate between the damaged and undamaged states of the structure based on the first three AR coefficients. Localization of damage was achieved by introducing another feature, also based on the AR coefficients, found to increase from a baseline value when damage was near. Nair and Kiremidjian ${ }^{13}$ investigated Gaussian Mixture Modeling, an unsupervised pattern recognition technique, to model the first AR coefficients obtained from fitting ARMA time series to an analytical, 12-DOF model of the ASCE Phase II Experimental SHM Benchmark Structure. The extent of damage was shown to correlate well with the Mahalanobis distances between undamaged and damaged feature clusters.

In order to differentiate between undamaged and damaged systems and locate and quantify damage analytical techniques are required to interpret patterns in the damage sensitive features. Such methods can broadly be divided into supervised techniques when data from damaged structures is available for training of pattern recognition algorithms, and unsupervised when it is not. The present study utilizes the supervised techniques of Nearest Neighbor Classification (NNC) and Learning Vector Quantization (LVQ), and unsupervised Self-organizing Maps (SOM). These algorithms have been applied in the past in a number of damage detection studies mostly concerned with faults in composite materials, mechanical systems and aerospace structures.

Philippidis et al. ${ }^{14}$ classified the waveforms of acoustic emission signals recorded during destructive tensile tests on coupons of composite materials using LVQ. Raju Damarla et al. ${ }^{15}$ and Doyle and Fernando ${ }^{16}$ analyzed high frequency vibration data from damaged composite panels and classified damage using frequency and time domain features presented to back-propagation (BP) and LVQ artificial neural network (ANN). Tse et al. ${ }^{17}$ studied a tapping machine using statistical features of dynamic forces and torques measured in the various machine components and identified several states in which the machine was operating using the LVQ algorithm. Abu-Mahfouz ${ }^{18}$ considered classification of damage in a gearbox system combining frequency response data and basic statistical measures of time-domain dynamic signals comparing BP, functional link and LVQ networks. Trendafilova et al. ${ }^{19}$ considered detection of damage by examining frequency response functions of the FEM model of an aircraft wing using the NNC methodology.

Jammu and Danai ${ }^{20}$ developed a single category based classifier using the SOM concept that required data only from normal conditions of machining equipment. Faults were detected by comparing distances to new feature samples to a predefined threshold. Leem ${ }^{21}$ proposed using a SOM for clustering of signals corresponding to levels of wear in machining equipment. Spectral points of force and acoustic emission signals as well as cutting speed and feed rate were used as damage features. Huguet et al. ${ }^{22}$ performed experimental work on coupons of composite material and used acoustic emission signals to identify various damage types. The use of a non-linear projection of SOM helped to visualize the topology of feature space. Godin et al. ${ }^{23}$ examined acoustic waveforms from progressively damaged composite materials to differentiate between different damage mechanisms and monitor damage chronology. They compared the supervised k-nearest neighbor (k-NN) algorithm and unsupervised SOM and found similar performance but k-NN was easier to use. Qui et al. ${ }^{24}$ applied a wavelet filter and SOM for performance assessment of bearing rolling element. The wavelet filter was used for signal denoising and enhancing of weak signatures and SOM for assessment of bearing degradation utilizing time domain features. Huang et al. ${ }^{25}$ studied prediction of ball bearing useful life using experimental data and SOM for detecting degradation using several time and frequency domain features. Casoetto et al ${ }^{26}$ established a feature map using peaks in the power spectral density for normal operation of a welding electrode via unsupervised learning. Degradation of the electrode was monitored by observing feature migration from the normal state. De Oliveira and Marquez ${ }^{27}$ clustered acoustic emission signals from composite samples in tensile tests using SOM and subsequently used k-means algorithm for damage classification. Bar et al. ${ }^{28}$ evaluated a combination of SOM and multilayer perceptron (MLP) for clustering of acoustic emission signals from tensile tests on composites. The SOM was used first to classify information for different failure modes, which was later used as information for training MLP for automatic classification. Yang et al. ${ }^{29}$ achieved damage classification by analyzing vibration signals from compressors using statistical moments of wavelet coefficients classified using LVQ, SOM and radial basis function networks (RBF).

7647 - 171 V. 2 (p.2 of 12) / Color: No / Format: A4 / Date: 2010-01-20 12:13:00 AM

SPIE USE: DB Check, Prod Check, Notes: 
SOM achieved a better performance than LVQ but RBF surpassed both of them. Mujica et al. ${ }^{30}$ proposed an adaptable case-based reasoning (CBR) approach to damage detection. Dynamic signals were processed via wavelet transform and wavelet coefficients used as damage features. A SOM was trained to organize available damage cases in memory and CBR used to obtain diagnostics by analogy.

Despite the interest the discussed pattern recognition techniques drew amongst the composite materials, machine and aircraft monitoring community, the application of NNC and LVQ for classification of damage to civil infrastructure has only recently been investigated ${ }^{31}$ and to the best of the authors' knowledge SOM has not yet been applied. This study develops a method for structural damage detection that integrates the use of AR models to establish damage sensitive features and application of pattern recognition techniques for classification of the AR coefficients depending on damage type and location. NNC, LVQ and SOM approaches to pattern recognition are compared. The proposed method is tested on two experimental structures.

\section{OUTLINE OF DAMAGE CLASSIFICATION METHOD AND UNDERLYING THEORY}

\subsection{Outline of damage classification method}

The proposed approach to damage classification can be summarized as follows:

- Structural acceleration time histories from multiple sensors in the undamaged and various damaged states are fitted with univariate AR models.

- The coefficients of AR models, arranged in a vector, are used as damage sensitive feature.

- If required, the reduction of the dimensionality of damage sensitive feature is performed using Principal Component Analysis (PCA) or Sammon mapping.

- LVQ or SOM pattern recognition algorithm is trained to classify the (possibly reduced) AR coefficients into a specific damage state. (Note NNC does not require training.)

- NNC, LVQ or SOM is used to classify the (possibly reduced) AR coefficients into a specific damage state. The AR coefficient vectors are fed into the pattern recognition algorithms as individual vectors even though several of them may belong to the same experiment.

The following sections describe briefly the underlying theory of techniques used in this study: AR time series models, PCA, Sammon mapping, NNC, LVQ and SOM.

\subsection{Autoregressive models}

In this research, AR time series models are used to describe the acceleration time histories. AR models are a staple of time series analysis ${ }^{32}$ and are often used in the analysis of stationary time series processes. A stationary process is a stochastic process, one that obeys probabilistic laws, in which the mean, variance and higher order moments are time invariant. AR models attempt to account for the correlations of the current observation in time series with its predecessors. A univariate $\mathrm{AR}$ model of order $p$, or $\operatorname{AR}(p)$, for the time series $\left\{x_{t}\right\}(t=1,2, \ldots, n)$ can be written as:

$$
x_{t}=\phi_{1} x_{t-1}+\phi_{2} x_{t-2}+\ldots+\phi_{p} x_{t-p}+a_{t}
$$

where $x_{t}, \ldots x_{t-p}$ are the current and previous values of the series, $\phi_{1}, \ldots \phi_{p}$ are AR coefficients, and $\left\{a_{t}\right\}$ is a Gaussian white noise error time series with a zero mean.

\subsection{Principal Component Analysis}

PCA is a popular multivariate statistical technique often used to reduce multidimensional data sets to lower dimensions ${ }^{33}$. Given a set of $p$-dimensional vectors $\mathbf{x}_{i}(i=1, \ldots, n)$ drawn from a statistical distribution with mean $\overline{\mathbf{x}}$ and covariance matrix $\Sigma$, PCA seeks to project the data onto a new $p$-dimensional space with orthogonal coordinates via a linear transformation.

Decomposition of the covariance matrix by singular value decomposition leads to:

$$
\Sigma=\mathbf{V} \Lambda \mathbf{V}^{T}
$$


where $\Lambda$ is a diagonal matrix containing the eigenvalues of $\Sigma$ ranked in the descending order $\sigma_{1}^{2} \geq \ldots \geq \sigma_{p}^{2}$, and $\mathbf{V}$ is a matrix containing the corresponding eigenvectors or principal components. The transformation of a data point $\mathbf{x}_{i}$ into principal components is:

$$
\mathbf{z}_{i}=\mathbf{V}^{T}\left(\mathbf{x}_{i}-\overline{\mathbf{x}}\right)
$$

The new coordinates $\mathbf{z}_{i}$ are uncorrelated and have a diagonal covariance matrix $\Lambda$. Therefore, the entries of $\mathbf{z}_{i}$ are linear combinations of the entries of $\mathbf{x}_{i}$ which explain variances $\sigma_{1}^{2}, \ldots, \sigma_{p}^{2}$. To reduce the dimensionality, a selection $q<p$ of principal components can be used that retains those components that contribute most to the data variance, thus reducing the dimension of the data to $q$.

\subsection{Sammon mapping}

Sammon mapping ${ }^{34}$ is a nonlinear transformation used for mapping a high dimensional space to a lower dimensional space in which local geometric relations are approximated. Consider a set of vectors $\mathbf{x}_{i}(n=1, \ldots, n)$ in a $p$-dimensional space and a corresponding set of vectors $\mathbf{y}_{i}$ in a $q$ - dimensional space, where $q<p$. For visualization purposes $q$ is usually chosen to be two or three. The distance between vectors $\mathbf{x}_{i}$ and $\mathbf{x}_{j}$ in $p$-dimensional space is denoted by $D_{i j}^{*}$ and the distance between the corresponding vectors $\mathbf{y}_{i}$ and $\mathbf{y}_{j}$ in $q$-dimensional space is $D_{i j}$. Usually the Euclidean distance is used. Mapping is achieved by adjusting the vectors $\mathbf{y}_{i}$ to minimize the following error function by steepest descent:

$$
E=\frac{1}{\sum_{i=1}^{n} \sum_{j<i} D_{i j}^{*}} \sum_{i=1}^{n} \sum_{j<i} \frac{\left(D_{i j}-D_{i j}^{*}\right)^{2}}{D_{i j}^{*}}
$$

\subsection{Nearest Neighbor Classification}

NNC is a simple supervised pattern recognition technique ${ }^{35}$. Given a set of pre-selected and fixed reference or codebook vectors $\mathbf{m}_{i}(i=1, \ldots, k)$ corresponding to known classes, an unknown input vector $\mathbf{x}$ is assigned to the class which the nearest $\mathbf{m}_{i}$ belongs. Several distance measures can be used including Manhattan, Euclidean, Correlation and Mahalanobis. In this research the Euclidean and Mahalanobis distance measures were used. The Euclidean $D_{E}(\mathbf{x}, \mathbf{y})$ and Mahalanobis distance $D_{M}(\mathbf{x}, \mathbf{y})$ between two vectors $\mathbf{x}$ and $\mathbf{y}$ can be calculated using:

$$
D_{E}(\mathbf{x}, \mathbf{y})=\sqrt{(\mathbf{x}-\mathbf{y})^{T}(\mathbf{x}-\mathbf{y})}, \quad D_{M}(\mathbf{x}, \mathbf{y})=\sqrt{(\mathbf{x}-\mathbf{y})^{T} \Sigma^{-1}(\mathbf{x}-\mathbf{y})}
$$

where $\Sigma$ is the covariance matrix of a distribution. The Mahalanobis distance accounts explicitly for the different scales and correlations amongst vector entries and can be more useful in cases where these are significant.

\subsection{Learning Vector Quantization}

LVQ is a supervised machine learning technique designed for classification or pattern recognition by defining class borders $^{35}$. It is similar to NNC in that it uses a set of codebook vectors and searches for the minimum of distances of an unknown vector to these codebook vectors as the criterion for classification. However, unlike in NNC where codebook vectors are fixed, an iterative procedure is adopted in which the position of the codebook vectors is adjusted to minimize the number of misclassifications. Learning of the optimal codebook vector positions can be performed using several algorithms. In this study, the Optimized-Learning-Rate LVQ1 ${ }^{35}$ algorithm was used. This algorithm has an individual learning rate for each codebook vector, resulting in faster training.

Given a set of initial codebook vectors $\mathbf{m}_{i}(i=1, \ldots, k)$ which have been linked to each class region, the input vector $\mathbf{x}$ is assigned to the class which the nearest $\mathbf{m}_{i}$ belongs, i.e. an NNC task is performed. Let $c$ define the index of the nearest codebook vector, i.e. $\mathbf{m}_{c}$. Learning is an iterative procedure in which the position of the codebook vectors is adjusted to minimize the number of misclassifications. At iteration step $t$ let $\mathbf{x}(t)$ and $\mathbf{m}_{i}(t)$ be the input vector and codebook vectors respectively. The $\mathbf{m}_{i}(t)$ are adjusted according to the following learning rule:

$$
\begin{aligned}
& \mathbf{m}_{c}(t+1)=\mathbf{m}_{c}(t)+\boldsymbol{\alpha}_{c}(t)\left[\mathbf{x}(t)-s(t) \mathbf{m}_{c}(t)\right] \\
& \mathbf{m}_{i}(t+1)=\mathbf{m}_{i}(t) \text { for } i \neq c
\end{aligned}
$$


where $s(t)$ equals +1 or -1 if $\mathbf{x}(t)$ has been respectively classified correctly or incorrectly, and $\boldsymbol{\alpha}_{c}(t)$ is the variable learning rate for codebook vector $\mathbf{m}_{c}$ :

$$
\boldsymbol{\alpha}_{c}(t)=\frac{\boldsymbol{\alpha}_{c}(t-1)}{1+s(t) \boldsymbol{\alpha}_{c}(t-1)}
$$

The learning rate must be constrained such that $\boldsymbol{\alpha}_{c}(t)<1$.

\subsection{Self-organizing Maps}

SOM is an unsupervised clustering technique that attempts to create low dimensional projections of high dimensional data in which the organizational structure, relative distances between adjacent data points is retained ${ }^{35}$. SOM is similar to LVQ in that the position of the codebook vectors is adjusted iteratively. However, SOM is an unsupervised learning algorithm in which adjustment is based on the similarity between input and codebook vectors only and class structure for the input is not required. Given a set of reference vectors $\mathbf{m}_{i}(i=1, \ldots, k)$ which have been initialized over the input space, the input vector $\mathbf{x}$ is assigned to the class which the nearest $\mathbf{m}_{i}$ belongs. Let $c$ define the index of the nearest reference vector, i.e. $\mathbf{m}_{c}$. During training the $\mathbf{m}_{i}$ are adjusted according to the iterative application of the following rule:

$$
\begin{aligned}
& \mathbf{m}_{c}(t+1)=\mathbf{m}_{c}(t)+\boldsymbol{\alpha}(t)\left[\mathbf{x}(t)-\mathbf{m}_{c}(t)\right] \\
& \mathbf{m}_{i}(t+1)=\mathbf{m}_{i}(t) \text { for } i \neq c
\end{aligned}
$$

Where $\alpha(t)$ must satisfy $\lim _{t \rightarrow \infty} \alpha(t)=0$ for convergence. In this investigation, $\alpha(l)=0.5 /(0.1 l+1)$ was adopted, where $l$ was the number of epochs, i.e. number of complete runs though the training data ${ }^{36}$.

\section{APPLICATION TO 3-STOREY LABORATORY BOOKSHELF STRUCTURE}

The 3-storey experimental bookshelf structure used in this study was approximately $2.1 \mathrm{~m}$ high and constructed from equal angle aluminum column sections and stainless steel floor plates bolted together with aluminum brackets as shown in Figure 1. The stainless steel plates were $4 \mathrm{~mm}$ thick and $650 \mathrm{~mm} \times 650 \mathrm{~mm}$ square. The column sections were $30 \mathrm{~mm} \times$ $30 \mathrm{~mm}$ equal angles. Two section thicknesses were used for the columns, either $3.0 \mathrm{~mm}$ or $4.5 \mathrm{~mm}$ for the damaged and undamaged states respectively. Each column was made of $3 \times 0.7 \mathrm{~m}$ high segments, rather than one long angle, in order to make them easily replaceable for simulation of localized damage at different stories. The structure was instrumented with four uniaxial accelerometers, one for measuring the table acceleration and one for each storey.

Damage was introduced into the structure by replacing the original $4.5 \mathrm{~mm}$ thick columns of a particular storey with $3.0 \mathrm{~mm}$ angles. Four damage states were considered; these were labeled D0, D1, D2 and D3 corresponded to no damage (healthy structure), $1^{\text {st }}$ storey damage, $2^{\text {nd }}$ storey damage and simultaneous $1^{\text {st }}$ and $2^{\text {nd }}$ story damage. Figure 2 schematically shows these four damage states together with the percentage of remaining lateral stiffness.

The objective of the damage detection study was to classify seismic responses measured in the four damage states and to that end eight scaled earthquake records were used to excite the structure. The acceleration time history of each storey was modeled using a univariate $\mathrm{AR}(24)$ model.

\subsection{Damage classification in the 3-storey bookshelf structure}

Using three univariate $\mathrm{AR}(24)$ models, one for each floor, resulted in 72-dimensional vectors of damage sensitive features. As a preliminary investigation, to visualize and check the presence of clusters in the data, PCA and Sammon mapping were used to create two and three-dimensional projections of the vectors of AR coefficients. The result of the two-dimensional projection using Sammon mapping is shown in Figure 3. The Sammon map shows some organization of the data into overlapping bands, although no distinct clusters could be drawn. Using three-dimensional mappings did not provide a better separation and PCA projection showed no clearly defined clusters with data points from all four damage states scattered amongst one another. These preliminary insights indicate that higher dimensional data need to be investigated to achieve separation of the AR coefficients from different damage states. Simple visual techniques will then be inadequate and more advanced approaches such as NNC, LVQ or SOM are required.

The two previously described pattern recognition techniques, NNC and LVQ were first used to classify damage into the states D0-D3. PCA data reduction techniques only was used due to the markedly higher computational effort required for Sammon mapping.

7647 - 171 V. 2 (p.5 of 12) / Color: No / Format: A4 / Date: 2010-01-20 12:13:00 AM 
a)

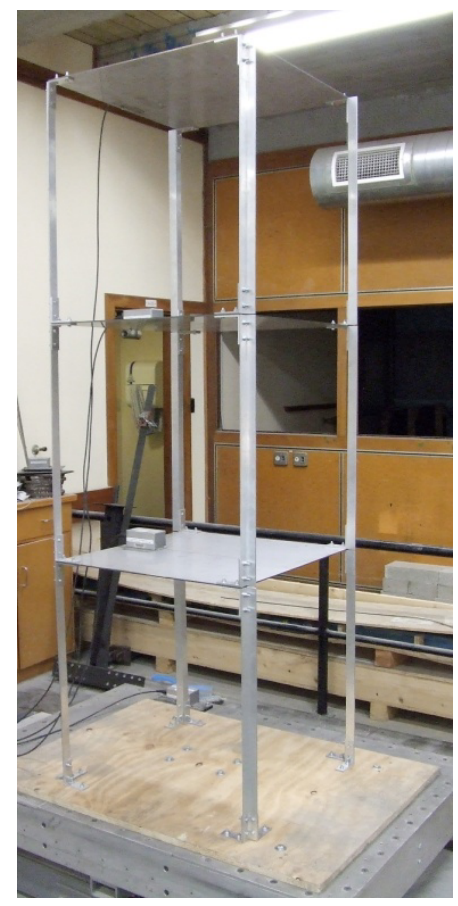

b)

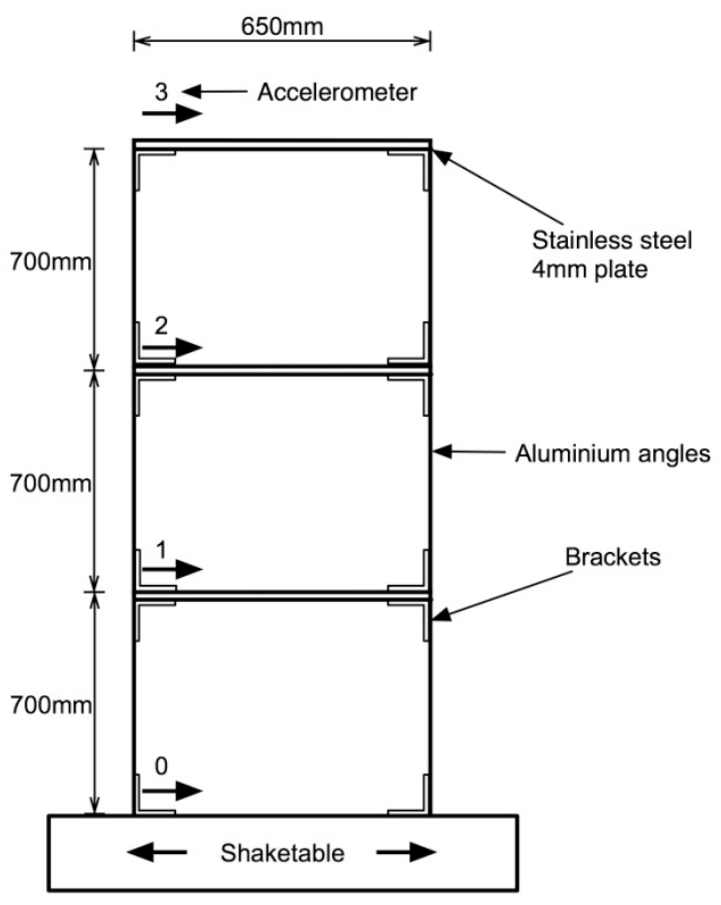

Figure 1. Three-storey bookshelf structure: (a) general view, and (b) dimensions and accelerometer locations.

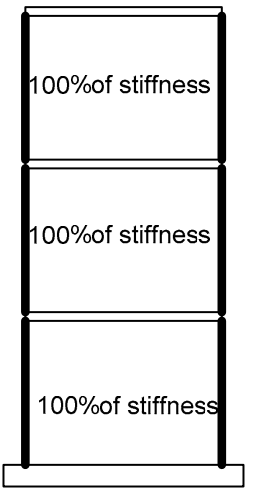

D0

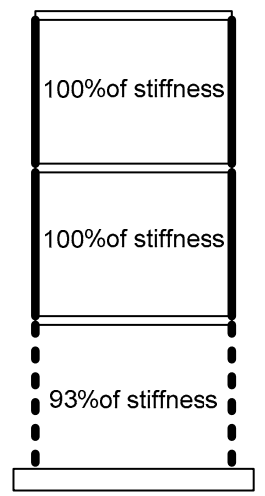

D1

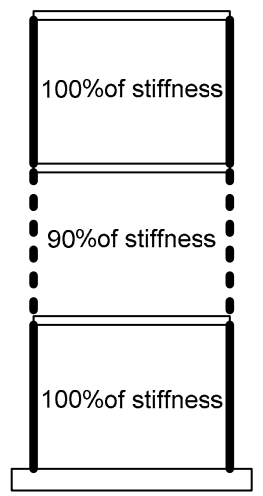

D2

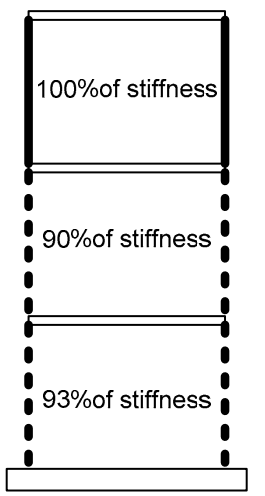

Legend:

$4.5 \mathrm{~mm}$ thick angle

:3.0mm thick angle

Figure 2. Damage states in 3-storey bookshelf structure showing percentage of lateral stiffness at each storey.

The feature dimension was reduced to the first $60,40,30,20$ or 10 principal components. The 388-point data set, consisting of 97 points from each damage state was randomly divided into 300 codebook vectors and 88 testing points, ensuring approximately equal numbers of damage features from each damage state (for the testing data these numbers were 21 for state D0, 23 for state D1, 21 for state D2, and 23 for state D3, respectively). Five different random sets of codebook vectors were considered. Using NNC and averaging the results from five runs, the obtained number of misclassifications and percentage errors is given in Table 1 for both the Euclidean and Mahalanobis distance measures. The Mahalanobis distance measure outperformed the Euclidean by a considerable margin and adequate results with $6 \%$ misclassifications were obtained using 20 principal components. Good classification results, 5\% or less misclassifications, were achieved using more than 30 principal components, while excellent classification, $1 \%$ or less misclassifications, required 60 principal components. Using the Euclidean distance measure a large number of 


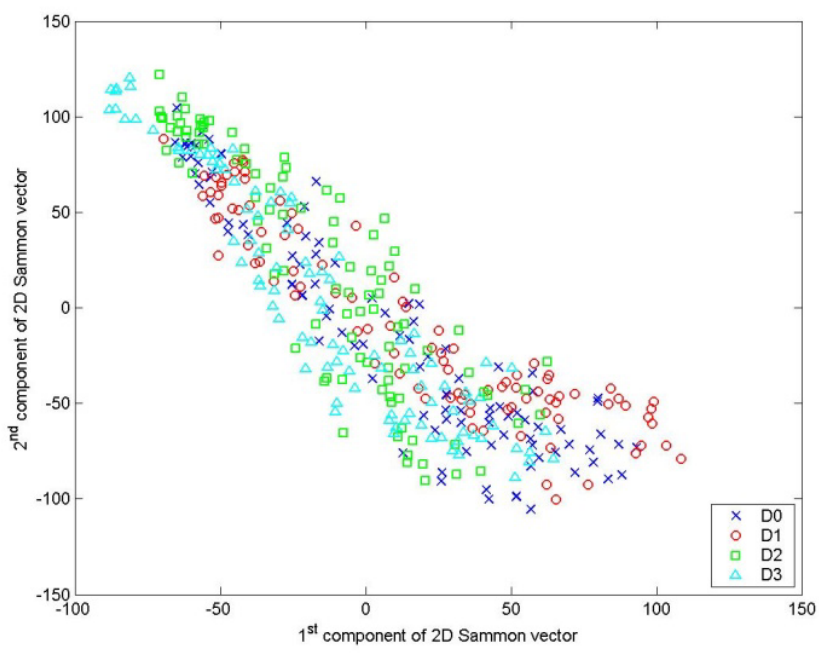

Figure 3. Projection of data from 3-storey bookshelf structure via Sammon mapping.

misclassifications were obtained, in excess of $34 \%$ misclassifications. The difference in performance between the Mahalanobis and Euclidean distance measures could be explained by the fact that the Mahalanobis distance accounts for the different scales of each principal component.

Although NNC performed well, performance could be improved by using a more advanced classification technique such as LVQ. The LVQ classification was used with the Mahalanobis distance measure and the PCA dimensionality reduction technique. The 388-point data set was again divided into 300 points for training and 88 points for testing. The number of codebook vectors was chosen to be 30,50 or 100 . These were initialized by random selection from the training data set. The results averaged from five runs with different initialized codebook vectors are shown in Table 2 . The table shows that increasing the number of codebook vectors reduced the number of misclassifications. Good classifications were obtained when 20 or more principal components were used while excellent classification was achieved using 30 principal components. Overall, LVQ performed better than NNC with excellent classifications obtained using 30 principal components and much smaller numbers of codebook vectors compared to 60 principal components using NNC.

Preliminary attempts to use SOM on the data from the 3-storey experimental bookshelf structure failed to deliver satisfactory results.

\section{APPLICATION TO ASCE PHASE II EXPERIMENTAL SHM BENCHMARK STRUCTURE}

The ASCE Phase II Experimental SHM Benchmark Structure ${ }^{37}$ is a 4-storey 2-bay by 2-bay steel frame with a $2.5 \mathrm{~m} \times$ $2.5 \mathrm{~m}$ floor plan and a height of $3.6 \mathrm{~m}$ (Figure 4). The columns are B100×9 sections and the floor beams are $\mathrm{S} 75 \times 11$

Table 1. Number and percentage of misclassifications using NNC and PCA reduced data for 3-storey bookshelf structure.

\begin{tabular}{|c|c|c|}
\hline $\begin{array}{c}\text { Number of } \\
\text { principal } \\
\text { components }\end{array}$ & $\begin{array}{c}\text { Euclidean } \\
\text { distance }\end{array}$ & $\begin{array}{c}\text { Mahalanobis } \\
\text { distance }\end{array}$ \\
\hline 60 & $31(35 \%)$ & $1(1 \%)$ \\
\hline 40 & $34(39 \%)$ & $3(3 \%)$ \\
\hline 30 & $30(34 \%)$ & $4(5 \%)$ \\
\hline 20 & $34(39 \%)$ & $5(6 \%)$ \\
\hline 10 & $34(39 \%)$ & $10(11 \%)$ \\
\hline
\end{tabular}

Table 2. Number and percentage of misclassifications using LVQ and PCA reduced data for 3-storey bookshelf structure.

\begin{tabular}{|c|c|c|c|}
\hline $\begin{array}{c}\text { Number of } \\
\text { principal } \\
\text { components }\end{array}$ & \multicolumn{3}{|c|}{ Number of codebook vectors } \\
\cline { 2 - 4 } & 30 & 50 & 100 \\
\hline 30 & $0(0 \%)$ & $0(0 \%)$ & $0(0 \%)$ \\
\hline 20 & $4(5 \%)$ & $3(3 \%)$ & $3(3 \%)$ \\
\hline 10 & $17(19 \%)$ & $15(17 \%)$ & $14(16 \%)$ \\
\hline
\end{tabular}




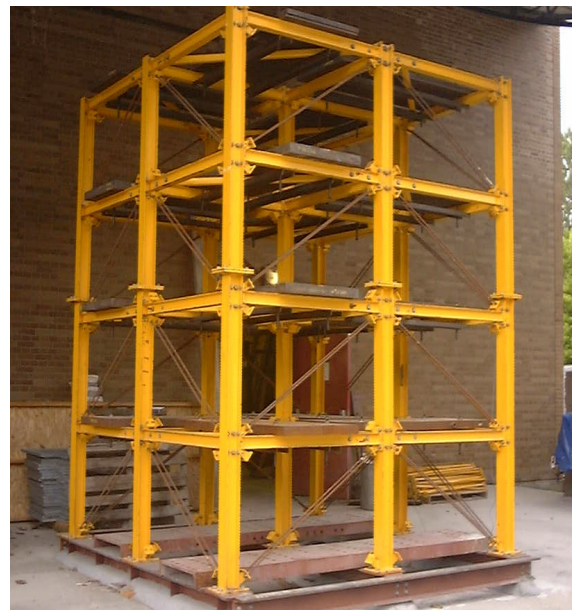

Figure 4. ASCE Phase II Experimental SHM Benchmark Structure.

Table 3. Damage configurations for ASCE Phase II Experimental SHM Benchmark Structure.

\begin{tabular}{|c|l|}
\hline Configuration & \multicolumn{1}{|c|}{ Damage } \\
\hline 1 & No damage \\
\hline 2 & All bracing removed on the E face \\
\hline 3 & Bracing removed on floors 1-4 on a bay on the SE corner \\
\hline 4 & Bracing removed on floors 1 and 4 on a bay on the SE corner \\
\hline 5 & Bracing removed on floors 1 on a bay on the SE corner \\
\hline 6 & All bracing removed on E face and floor 2 on N face \\
\hline 7 & All bracing removed \\
\hline 8 & Configuration 7 + loosened bolts on floors 1-4 on E face N bay \\
\hline 9 & Configuration 7 + loosened bolts on floors 1 and 2 on E face N bay \\
\hline
\end{tabular}

sections, all sections are Grade 300 steel. The beams and columns are bolted together. Bracing was added in all bays with two $12.7 \mathrm{~mm}$ diameter threaded steel rods. Additional mass was distributed around the structure to make it more realistic. Four $1000 \mathrm{~kg}$ floor slabs were placed on the $1^{\text {st }}, 2^{\text {nd }}$ and $3^{\text {rd }}$ floors, one per bay. On the $4^{\text {th }}$ floor, four $750 \mathrm{~kg}$ slabs were used. Two of the slabs per floor are placed off-centre to increase the coupling between translational and torsional motion.

A total of 9 damage scenarios were simulated on the structure, these involved the removal of bracing and the loosening of bolts in the floor beam connections. Table 3 lists the damage states and gives a description of damage. The different configurations give a mixture of minor and extensive damage cases. The structure was instrumented with 15 accelerometers and excited by a shaker with a band-limited $5-50 \mathrm{~Hz}$ white noise. Univariate AR(30) models were adopted and fitted to the acceleration data from each of the 15 accelerometers. This resulted in the dimension of feature (AR coefficients) vectors of 450 . A data set of 1035 feature vectors was obtained, 115 from each configuration.

\subsection{Damage classification in the ASCE Phase II Experimental SHM Benchmark Structure}

Preliminary investigations showed that using Sammon mapping, see Figure 5, six large-scale clusters could be seen. Two of the clusters consisted of configurations 1,5 and 6, and configurations 3 and 4 with small amount of overlapping between these configurations, while the remaining clusters were solely formed by a single configuration.

For the purpose of damage classification using $\mathrm{NNC}$, the feature dimension was reduced by projection of the data onto the first 20,10, 5 or 3 principal components. The 1035-point data set was randomly divided into 700 codebook vectors and 335 testing points, ensuring approximately equal numbers of damage features from each damage state (for the testing data these numbers were $39,36,35,39,40,36,37,32$ and 31 for damage configurations 1 through 9 , respectively). Averaging the results from five runs with different selection of codebook vectors the number of misclassifications and 


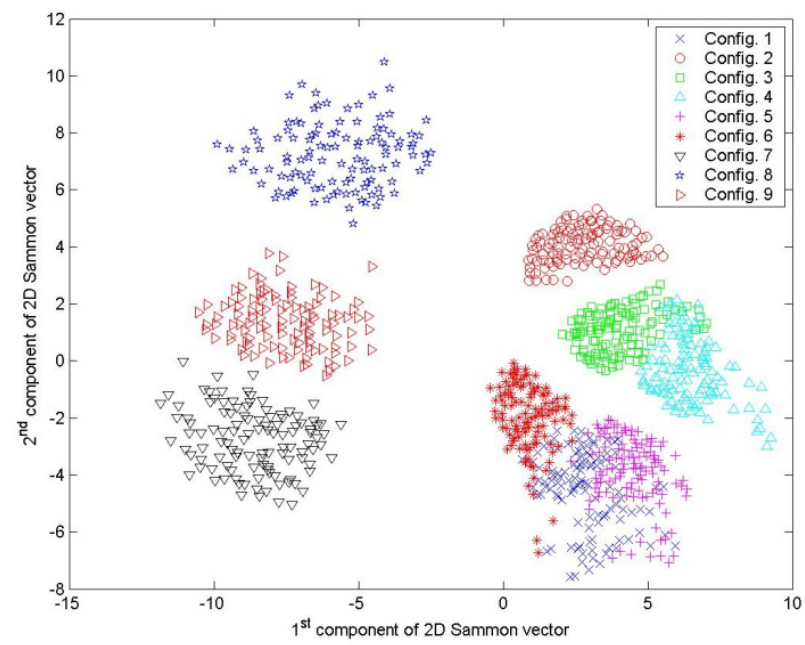

Figure 5. Projection of data from ASCE Phase II Experimental SHM Benchmark Structure via Sammon mapping.

percentage errors is given in Table 4. In this case, similar performance was obtained using both the Euclidian and Mahalanobis distance measures. This can be attributed to the fact that the data from different damage configuration seems to be well separated. Excellent performance was obtained using only 10 principal components. These results represent significant reduction in dimensionally while good accuracy was maintained.

LVQ classification was applied to PCA-reduced data with the same number of principal components as previously and the same sized training and testing data sets were used. The results from NNC showed that performance was similar for both distance measures, and hence only the Euclidean distance was chosen for LVQ. The number of codebook vectors was either 50, 100 or 200. These were initialized by random selection from the training set. The results obtained from averaging the number of misclassifications from five runs are shown in Table 5. The table shows that increasing the number of codebook vectors generally resulted in better performance. Excellent performance was obtained using 20 principal components for 100 and 200 codebook vectors. Good classification was still achieved using 10 principal components, however, errors became significant once fewer principal components were used. Overall, performance was slightly worse than NNC but achieved with significantly smaller numbers of codebook vectors.

Unlike in the case of the 3-storey bookshelf structure data, Figure 5 clearly showed the presence of distinct damage clusters corresponding to specific damage configurations. This encouraged an attempt to use SOM for unsupervised damage classification. Although, SOM was not designed for supervised classification tasks, provided the true classification is known the number of points misclassified to each cluster is an appropriate measure of performance. A

Table 4. Number and percentage of misclassifications using NNC and PCA reduced data for ASCE Phase II Experimental SHM Benchmark Structure.

\begin{tabular}{|c|c|c|}
\hline $\begin{array}{c}\text { Number of } \\
\text { principal } \\
\text { components }\end{array}$ & $\begin{array}{c}\text { Euclidean } \\
\text { distance }\end{array}$ & $\begin{array}{c}\text { Mahalanobis } \\
\text { distance }\end{array}$ \\
\hline 20 & $1(0.3 \%)$ & $1(0.3 \%)$ \\
\hline 10 & $5(1 \%)$ & $5(1 \%)$ \\
\hline 5 & $23(7 \%)$ & $24(7 \%)$ \\
\hline 3 & $62(19 \%)$ & $65(19 \%)$ \\
\hline
\end{tabular}

Table 5. Number and percentage of misclassifications using LVQ and PCA reduced data for ASCE Phase II Experimental SHM Benchmark Structure.

\begin{tabular}{|c|c|c|c|}
\hline $\begin{array}{c}\text { Number of } \\
\text { principal } \\
\text { components }\end{array}$ & \multicolumn{3}{|c|}{ Number of codebook vectors } \\
\cline { 2 - 4 } & 50 & 100 & 200 \\
\hline 20 & $6(2 \%)$ & $5(1 \%)$ & $4(1 \%)$ \\
\hline 10 & $13(4 \%)$ & $10(3 \%)$ & $6(2 \%)$ \\
\hline 5 & $24(7 \%)$ & $29(9 \%)$ & $23(7 \%)$ \\
\hline 3 & $75(22 \%)$ & $68(20 \%)$ & $67(20 \%)$ \\
\hline
\end{tabular}


Table 6. Damage classification using SOM and Sammon mapping reduced data for ASCE Phase II Experimental SHM Benchmark Structure

\begin{tabular}{|c|c|c|c|c|c|c|c|c|c|}
\hline & \multicolumn{10}{|c|}{ Cluster } \\
\cline { 2 - 11 } Configuration & 1 & 2 & 3 & 4 & 5 & 6 & 7 & 8 & 9 \\
\hline 1 & 34 & 0 & 0 & 0 & 75 & 6 & 0 & 0 & 0 \\
\hline 2 & 0 & 115 & 0 & 0 & 0 & 0 & 0 & 0 & 0 \\
\hline 3 & 0 & 0 & 115 & 0 & 0 & 0 & 0 & 0 & 0 \\
\hline 4 & 3 & 0 & 0 & 112 & 0 & 0 & 0 & 0 & 0 \\
\hline 5 & 12 & 0 & 0 & 0 & 103 & 0 & 0 & 0 & 0 \\
\hline 6 & 11 & 0 & 0 & 0 & 0 & 104 & 0 & 0 & 0 \\
\hline 7 & 0 & 0 & 0 & 0 & 0 & 0 & 114 & 0 & 1 \\
\hline 8 & 0 & 0 & 0 & 0 & 0 & 0 & 0 & 113 & 2 \\
\hline 9 & 0 & 0 & 0 & 0 & 0 & 0 & 111 & 0 & 4 \\
\hline
\end{tabular}

10D Sammon map was constructed and cluster analysis was performed on the reduced data using SOM with 9 reference vectors randomly initialized over the input space and all 1035 available damage features. The classification results have been shown in Table 6 in which the assigned cluster and true damage configuration has been given. Clusters corresponding to damage configurations 2 and 3 were $100 \%$ correctly classified while configurations 4,7 and 8 gave good classifications with between $97 \%$ and $99 \%$ of success. Configuration 9 was poorly classified with only $3 \%$ correct. The results show that a total of 221 misclassifications or $21 \%$ misclassifications were present, the misclassification of samples from configuration 9 to 7 alone responsible for half of the total error. This is a rather surprising outcome, as from Figure 5 these two configurations appear to be very well separated, and requires further assessment. Compared to supervised classification the result were generally worse as a consequence of providing the classification algorithm with less information but still quite promising.

\section{CONCLUSIONS}

SHM and damage detection methods can benefit from the applications of time series analysis techniques, which were developed for understanding long, regularly sampled sequences of data. In this study, a damage detection and classification method using AR models and NNC, LVQ or SOM statistical pattern recognition algorithm has been developed and applied to a simple 3-storey laboratory bookshelf structure and more complex ASCE Phase II Experimental SHM Benchmark Structure. Acceleration time histories of the structures in different simulated damage cases and under dynamic excitation were recorded and fitted using AR models. The coefficients of these AR models were chosen as damage sensitive features. Dimensionality reduction of the damage sensitive feature was achieved via PCA and Sammons mapping and served the purpose of visualization of clusters amongst the AR coefficients and lessening computational burden of the pattern recognition techniques. Systematic damage classification was studied using the NNC and LVQ supervised learning algorithms with either Euclidian or Mahalanobis distance measures, and unsupervised SOM algorithm.

The studies on the 3-storey laboratory structure demonstrated that for localized stiffness reduction of $7 \%$ to $10 \%$ the AR coefficient corresponding to different damage states, when projected on two dimensions, do not form clearly separable clusters. However, when the number of principal components used was increased to 60 and 30 respectively, both NNC and LVQ with the Mahalanobis distance measure were able to classify damage with no more than approximately $1 \%$ of misclassifications. The Euclidian measure, on the other hand produced approximately 35\% misclassifications. For the data from the ASCE Phase II Experimental SHM Benchmark Structure much more clearly delineated clusters of AR coefficients were seen in two dimensions. In this case the performance of both distance measures was comparable. The results using NNC and LVQ showed that approximately $1 \%$ misclassifications could be achieved using 20 principal components.

Unsupervised classification using SOM was attempted for the 3-storey laboratory structure but did not yield satisfactory results. In contrast, the application of SOM to the ASCE Phase II Experimental SHM Benchmark Structure showed promising results with the majority of damage configurations correctly classified. However, a total of $21 \%$ of misclassifications was observed, the large overall error caused by selected few damage classifications. 
Overall, the results showed that AR coefficients perform well as damage sensitive features, NNC and LVQ are reliable tools for damage classification, and significant reductions in the data dimensionality could be achieved whilst maintaining good performance. SOM appears to be a promising approach but requires further study.

\section{ACKNOWLEDGMENT}

The authors would like to express their gratitude for the Earthquake Commission Research Foundation of New Zealand for their partial financial support of this research (Project No. UNI/535).

\section{REFERENCES}

1. Fassois, S. D. and Sakellariou, J. S., "Time-series methods for fault detection and identification in vibrating structures," Philosophical Transactions of the Royal Society A: Mathematical, Physical and Engineering Sciences 365 (1851), 411-448 (2007).

2. Sadeghi, M. H. and Fassois, S. D., "Reduced-dimensionality geometric approach to fault identification in stochastic structural systems," AIAA Journal 36 (12), 2250-2256 (1998).

3. Sakellariou, J. S. and Fassois, S. D., "Stochastic output error vibration-based damage detection and assessment in structures under earthquake excitation," Journal of Sound and Vibration 297 (3-5), 1048-1067 (2006).

4. Sakellariou, J. S. and Fassois, S. D., "Vibration based fault detection and identification in an aircraft skeleton structure via a stochastic functional model based method," Mechanical Systems and Signal Processing 22 (3), 557-573 (2008).

5. Sohn, H., Czarnecki, J. A. and Farrar, C. R., "Structural health monitoring using statistical process control," Journal of Structural Engineering 126 (11), 1356-1363 (2000).

6. Sohn, H., Farrar, C. R., Hunter, N. F. and Worden, K., Applying the LANL statistical pattern recognition paradigm for structural health monitoring to data from a surface-effect fast patrol boat, Los Alamos National Laboratory, Los Alamos (2001).

7. Gul, M. and Catbas, N. F., "Statistical pattern recognition for structural health monitoring using time series modeling: Theory and experimental verifications," Mechanical Systems and Signal Processing 23 (7), 21922204 (2009).

8. Omenzetter, P. and Brownjohn, J. M. W., "Application of time series analysis for bridge monitoring," Smart Materials and Structures 15 (1), 129-138 (2006).

9. Zheng, H. and Mita, A., "Two-stage damage diagnosis based on the distance between ARMA models and prewhitening filters," Smart Materials and Structures 16 (5), 1829-1836 (2007).

10. Zheng, H. and Mita, A., "Damage indicator defined as the distance between ARMA models for structural health monitoring," Structural Control and Health Monitoring 15 (7), 992-1005 (2008).

11. Zheng, H. and Mita, A., "Localized damage detection of structures subject to multiple ambient excitations using two distance measures for autoregressive models," Structural Health Monitoring 8 (3), 207-222 (2009).

12. Nair, K. K., Kiremidjian, A. S. and Law, K. H., "Time series-based damage detection and localization algorithm with application to the ASCE benchmark structure," Journal of Sound and Vibration 291 (1-2), 349-368 (2006).

13. Nair, K. K. and Kiremidjian, A. S., "Time series based structural damage detection algorithm using Gaussian mixtures modeling," Journal of Dynamic Systems, Measurement and Control, Transactions of the ASME 129 (3), 285-293 (2007).

14. Philippidis, T. P., Nikolaidis, V. N. and Anastassopoulos, A. A., "Damage characterization of carbon/carbon laminates using neural network techniques on ae signals," NDT \& E International 31 (5), 329-340 (1998).

15. Raju Damarla, R., Karpur, P. and Bhagat, P. K., "A self-learning neural net for ultrasonic signal analysis," Ultrasonics 30 (5), 317-324 (1992).

16. Doyle, C. and Fernando, G., "Detecting impact damage in a composite material with an optical fibre vibration sensor system," Smart Materials and Structures 7 (4), 543-549 (1998).

17. Tse, P., Wang, D. D. and Atherton, D., "Harmony theory yields robust machine fault-diagnostic systems based on learning vector quantization classifiers," Engineering Applications of Artificial Intelligence 9 (5), 487-498 (1996). 
18. Abu-Mahfouz, I. A., "A comparative study of three artificial neural networks for the detection and classification of gear faults," International Journal of General Systems 34 (3), 261-277 (2005).

19. Trendafilova, I., Cartmell, M. P. and Ostachowicz, W., "Vibration-based damage detection in an aircraft wing scaled model using principal component analysis and pattern recognition," Journal of Sound and Vibration 313 (3-5), 560-566 (2008).

20. Jammu, V. B. and Danai, K., "Unsupervised neural network for tool breakage detection in turning," Annals of CIRP 42 (1), 67-71 (1993).

21. Leem, C. S., "A practical monitoring strategy for machining process control," International Journal of Production Research 35 (4), 1051-1066 (1997).

22. Huguet, S., Godin, N., Gaertner, R., Salmon, L. and Villard, D., "Use of acoustic emission to identify damage modes in glass fibre reinforced polyester," Composites Science and Technology 62 (10-11), 1433-1444 (2002).

23. Godin, N., Huguet, S., Gaertner, R. and Salmon, L., "Clustering of acoustic emission signals collected during tensile tests on unidirectional glass/polyester composite using supervised and unsupervised classifiers," NDT \& E International 37 (4), 253-264 (2004).

24. Qiu, H., Lee, J., Lin, J. and Yu, G., "Robust performance degradation assessment methods for enhanced rolling element bearing prognostics," Advanced Engineering Informatics 17 (3-4), 127-140 (2003).

25. Huang, R., Xi, L., Li, X., Richard Liu, C., Qiu, H. and Lee, J., "Residual life predictions for ball bearings based on self-organizing map and back propagation neural network methods," Mechanical Systems and Signal Processing 21 (1), 193-207 (2007).

26. Casoetto, N., Djurdjanovic, D., Mayor, R., Ni, J. and Lee, J., "Multisensor process performance assessment through use of autoregressive modeling and feature maps," Journal of Manufacturing Systems 22 (1), 64-72 (2003).

27. De Oliveira, R. and Marques, A. T., "Damage mechanisms identification in FRP using acoustic emission and artificial neural networks," Proc. Materials Science Forum, 789-793 (2006).

28. Bar, H. N., Bhat, M. R. and Murthy, C. R. L., "Identification of failure modes in GFRP using PVDF sensors: Ann approach," Composite Structures 65 (2), 231-237 (2004).

29. Yang, B. S., Han, T., An, J. L., Kim, H. C. and Ahn, B. H., "Technical note: A condition classification system for reciprocating compressors," Structural Health Monitoring 3 (3), 277-284 (2004).

30. Mujica, L. E., Vehi, J., Rodellar, J. and Kolakowski, P., "A hybrid approach of knowledge-based reasoning for structural assessment," Smart Materials and Structures 14 (6), 1554-1562 (2005).

31. Omenzetter, P. and de Lautour, O. R., "Nearest neighbor and learning vector quantization classification for damage detection using time series analysis," Structural Control and Health Monitoring, in print (2010).

32. Wei, W. W. S., Time series analysis: Univariate and multivariate methods, 2nd ed, Pearson, Boston (2006).

33. Sharma, S., Applied multivariate techniques, Wiley, New York (1997).

34. Sammon, J. J. W., "Nonlinear mapping for data structure analysis," IEEE Transactions on Computers C-18 (5), 401409 (1969).

35. Kohonen, T., Self-organizing maps, Springer, Berlin (1997).

36. Kecman, V., Learning and soft computing, MIT Press, Cambridge, Massachusetts (2001).

37. ASCE Structural Health Monitoring Committee, http://cive.seas.wustl.edu/wusceel/asce.shm/. 